\title{
Paper-Based Electrophoretic Bioassay: Biosensing in Whole Blood Operating via Smartphone
}

\author{
Amadeo Sena-Torralba, ${ }^{\S}$ Ruslan Alvarez-Diduk, ${ }^{\S}$ Claudio Parolo, Helena Torné-Morató, \\ Alexander Müller, and Arben Merkoçi*
}

Cite This: Anal. Chem. 2021, 93, 3112-3121

Read Online

ACCESS I

Llll Metrics \& More

Article Recommendations

Supporting Information

ABSTRACT: Point-of-care (PoC) tests are practical and effective diagnostic solutions for major clinical problems, ranging from the monitoring of a pandemic to recurrent or simple measurements. Although, in recent years, a great improvement in the analytical performance of such sensors has been observed, there is still a major issue that has not been properly solved: the ability to perform adequate sample treatments. The main reason is that normally sample treatments require complicated or long procedures not adequate for deployment at the PoC. In response, a sensing platform, called paperbased electrophoretic bioassay (PEB), that combines the key characteristics of a lateral flow assay (LFA) with the sample treatment capabilities of electrophoresis is developed. In particular, the ability of PEB to separate different types of particles and to detect human

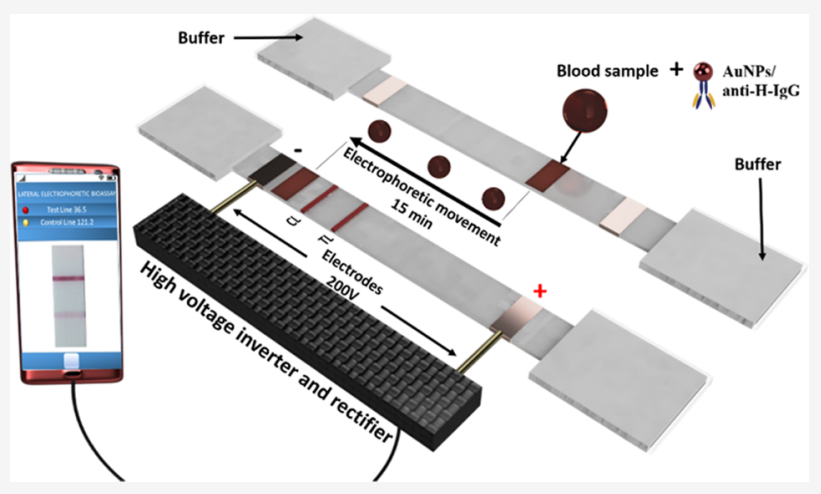
antibodies in untreated spiked whole blood is demonstrated. Finally, to make the platform suitable for PoC, PEB is coupled with a smartphone that controls the electrophoresis and reads the optical signal generated. It is believed that the PEB platform represents a much-needed solution for the detection of low target concentrations in complex media, solving one of the major limitations of LFA and opening opportunities for point-of-care sensors.

$\mathrm{T}$ he ability to measure a specific biomarker within minutes and in the easiest possible way is crucial for a variety of healthcare-related scenarios, ranging from the daily measurement of glucose level to the monitoring of a pandemic. ${ }^{1,2}$ In this context, laboratory-based technologies are not useful, considering that they require expensive/bulky equipment, long/multistep protocols, and qualified personnel. ${ }^{3,4}$ Instead, point-of-care (POC) tests represent a valid solution due to their ease of use, speed, low cost, and portability. ${ }^{5-8}$ In the realm of POC technologies, lateral flow assays (LFAs) stand out as the most widely used POC sensors across many disciplines, from environmental analysis to safety and diagnostics. Their success lies in their single-step procedure (the user has to just apply the sample and in some cases a washing buffer) and their ability to provide results within 1015 min. $^{9-11}$ The combination of their practicality with a low fabrication cost makes them a particularly attractive sensing platform for both developers and users. Indeed, the simple operation of LFAs is one of their major advantages over other sensing technologies, but it also comes with a major drawback: the inability to perform efficient sample treatments. This limitation of LFAs precludes their use for more challenging applications, for example, the detection of biomarkers at low concentrations in complex media.

Most commercially available LFAs are either single-step (i.e., pregnancy tests) or double-step (i.e., serological tests) assays.
The only sample treatment included in the former type of assays is the modification of the physical-chemical properties of the sample, thanks to the use of a prestored buffer (i.e., a buffer dried into the sample pad, which is the region of the test where the sample is applied). This allows for the stabilization of the $\mathrm{pH}$, ionic strength, and viscosity of the sample, which may be enough for clear samples with low viscosity (i.e., urine), but it is not sufficient for more viscous and complex matrixes (i.e., serum/blood). ${ }^{12}$ Regarding the latter type of LFAs, they can include either a dilution step (prior to the addition of the sample onto the sample pad) or a washing step (the addition of a washing buffer after the application of the sample). ${ }^{13,14}$ The washing buffer is usually based on a low ionic strength and neutral buffer doped with anionic surfactants such as Tween- $20^{15,16}$ or sodium dodecyl sulfate (SDS). ${ }^{12,17}$ These are frequently combined with external filtration units or integrated external filtration membranes, such as the commercial Cytosep and Vivid plasma separation

Received: October 14, 2020

Accepted: January 21, 2021

Published: February 3, 2021 
membranes. ${ }^{18-20}$ There are also publications reporting novel integrated plasma separation strategies. ${ }^{21,22}$ In this case, LFAs can also work with blood and serum samples, but it compromises their ease of use (i.e., the user has to dilute the sample, add a washing buffer or remove the filtration membrane at a fixed time) and possibly their sensitivity (the dilution of the sample implies a dilution of the target analyte). LFAs can also include reagents that remove specific contaminants (for example, antibodies for the removal of albumin), but this increases the complexity and cost of fabrication. ${ }^{23,24}$

Here, we propose a sensing platform that allows the use of whole blood while retaining all of the characteristics that make LFAs the most popular POC diagnostic technology. Particularly, we developed a paper-based electrophoretic bioassay (PEB) that uses the main components of LFAs, but instead of relying on the capillary force to move the sample, it uses electrophoresis. The use of a continuous and tunable flow to move the sample easily overcomes the main issues observed when using blood samples in LFAs, like nonspecific binding or the clogging of the strip. We validated the PEB using as a test bed the detection of Human Immunoglobulin G (H-IgG) in untreated spiked whole blood. Finally, we demonstrated the applicability of PEB at the point-of-care using a smartphonebased system that controls the electrophoresis and quantifies the optical signal.

\section{EXPERIMENTAL SECTION}

Materials, Reagents, and Instruments. Materials, reagents, and instruments, are detailed in the Supporting Information (SI).

PEB Device Design and Fabrication. A nitrocellulose (NC) membrane with a size of $10 \mathrm{~cm} \times 0.49 \mathrm{~cm}$ was used as a platform for running the electrophoresis. Two adhesive copper electrodes were stacked through their adhesive at $0.5 \mathrm{~cm}$ from both ends of the NC membrane. As stipulated by the manufacturer, both the copper and the adhesive provide low resistance to current and thus are suitable for their application as electrical connectors. Moreover, two cellulose pads $(6 \mathrm{~cm} \times$ $1.5 \mathrm{~cm}$ ) were used for the storage and gradual dispensing of the electrophoresis buffer and were stacked over the adhesive copper electrodes toward the extremes of the strip. The buffer is required to act as an electrolyte and maintain the $\mathrm{pH}$ at a relatively constant value. Next, the copper electrodes were connected to the power source system that contained all of the electronics inside a three-dimensional (3D)-printed polylactic acid (PLA) cartridge (see the electronic diagram in Figure S1 and the Results and Discussion section for more details). For the evaluation of the quantum dot (QD) mobility, a black cover was fabricated to measure the fluorescence emitted by the QDs. The cover was made of PLA and fabricated using a 3D printer. Two light-emitting diodes (LEDs) (365 nm excitation wavelength) and two white LEDs were placed inside the device cover and connected by an on-the-go (OTG) wire to a Smartphone (Figure S2).

Nitrocellulose Membrane Strip Fabrication. The NC membranes used for the evaluation of the electrophoresis performance were $\mathrm{CN} 95, \mathrm{CN} 110$, and $\mathrm{CN} 150$. For the detection of $\mathrm{H}-\mathrm{IgG}$, the $\mathrm{CN} 95 \mathrm{NC}$ membrane was used. Polyclonal goat to $\mathrm{H}-\mathrm{IgG}$ antibodies were deposited in the test line (TL) and polyclonal chicken to goat IgG antibodies were deposited in the control line (CL) (both at $1 \mathrm{mg} / \mathrm{mL}$ in 10 $\mathrm{mM}$ phosphate-buffered saline, PBS, $\mathrm{pH}$ 7.4) using a bioreagent dispenser. The antibodies were dried for $2 \mathrm{~h}$ at $37{ }^{\circ} \mathrm{C}$.

Evaluation of the Electrophoresis Performance. Commercial streptavidin-functionalized CdSe@ZnS QDs and $\mathrm{CdTe}$ QDs were used. The graphene quantum dots (G-QDs) were synthesized using a homemade method following the procedure of Dong et al. ${ }^{25}$ The nitrogen- and sulfur-doped GQDS (N-S-doped G-QDs) were synthesized following the procedure reported by Dong et al. ${ }^{26}$ The evaluation of the $\mathrm{pH}$ buffer effect on the electrophoretic mobility of the QDs was carried out by drop-casting $1 \mathrm{~mL}$ of sodium borate (SB) buffer on the cellulose pads. Buffer solutions at different $\mathrm{pH}$ values $(8,9$, and 10) were prepared using boric acid with sodium hydroxide and tested on different PEB strips working simultaneously. The gold nanoparticles (AuNPs) were synthesized by the Turkevich method ${ }^{27}$ using $50 \mathrm{~mL}$ of $\mathrm{HAuCl}_{4}(0.25 \mathrm{mM}$ in Milli-Q water $)$ and $1.25 \mathrm{~mL}$ of sodium citrate (1\% in Milli-Q water). They were conjugated with anti$\mathrm{H}-\mathrm{IgG}$ following the experimental procedure already reported by the group, ${ }^{28}$ detailed in the Supporting Information.

Detection of $\mathrm{H}$-IgG in SB Buffer and Whole Blood. One milliliter of SB buffer $(10 \mathrm{mM}, \mathrm{pH} 8)$ was drop-casted onto the cellulose pads. Once the entire strip was embedded with the electrophoresis buffer, the electrodes corresponding to the positive and negative charged poles were connected to the power source. Next, $3 \mu \mathrm{L}$ of the conjugated AuNPs was incubated with $3 \mu \mathrm{L}$ of chicken whole blood for $30 \mathrm{~s}$ and dropcasted onto the NC pad at $1 \mathrm{~cm}$ from the TL. Then, the power source was connected, and the assay was performed for $15 \mathrm{~min}$.

Evaluation of the PEB. Pictures of the strips were taken by adjusting the camera parameters of the smartphone (ISO 160, A 1/10, Manual focus) at a height distance of $9 \mathrm{~cm}$ from the strip and under constant light conditions, provided by the white LEDs. The pictures were evaluated using Image J software, following the procedure reported by our group. ${ }^{29}$ The signal intensity was normalized following the equation: $(\mathrm{TL}-\mathrm{BG}) /(\mathrm{CL}-\mathrm{BG})$, where $\mathrm{TL}, \mathrm{CL}$, and $\mathrm{BG}$ were the signal intensities of the test line, control line, and background. The limit of detection (LoD) was calculated as optical intensity $(\mathrm{LoD})=$ blank $+3 \sigma$ blank (i.e., the corresponding value of blank sample plus 3 times its standard deviation). The limit of quantification (LoQ) was calculated as optical intensity $($ LoQ $)=$ blank $+10 \sigma$ blank. $^{30}$

Detection of $\mathrm{H}$-IgG in Buffer and Whole Blood Using LFA. The CytoSep membrane was used as the sample pad and CN95 as the detection pad. The anti-H-IgG functionalized AuNP solution used in the conjugate pad was the same as the one used for the PEB experiments. The calibration curve for $\mathrm{H}-\mathrm{IgG}$ was performed by spiking $\mathrm{H}-\mathrm{IgG}(0-10000 \mathrm{ng} / \mathrm{mL})$ in PBS buffer (10 mM, pH 7.4). Then, $100 \mu \mathrm{L}$ of each solution were drop-casted onto the sample pad. For the calibration curve of H-IgG in whole blood, a solution of chicken whole blood spiked with $\mathrm{H}$-IgG $(1 \mu \mathrm{g} / \mathrm{mL})$ was prepared. The solution was diluted 2.4- and 5-fold using PBS buffer (10 mM, $\mathrm{pH}$ 7.4). Then, $100 \mu \mathrm{L}$ of the undiluted and diluted whole blood were drop-casted onto the sample pad. Pictures of the strips were taken after $15 \mathrm{~min}$ using the smartphone and adjusting the same camera parameters as for the PEB evaluation.

\section{RESULTS AND DISCUSSION}

Concept. Electrophoresis is the migration of charged particles under the influence of an electric field. The system 
is based on two electrodes of opposite charges (cathode and anode) connected by a conductive medium (electrolyte). The charged particles are able to separate due to differences in their velocity $(v)$, which depends on the particle's mobility $(m)$ and the applied field strength $(E)$ in such a way

$$
v=m E
$$

Moreover, the particle's mobility $(m)$ is determined by their size, shape, and charge and by the temperature during the separation. The mobility is constant under defined electrophoretic conditions, which are characterized both by electrical parameters (current, voltage, power) and factors related to the electrophoresis medium (ionic strength, $\mathrm{pH}$ value, viscosity, and pore size). In this sense, the electrophoretic mobility $(\mu)$ can be expressed as

$$
\mu=\frac{q}{6 \pi r \eta}
$$

where $q$ is the charge on the particle, $\eta$ is the viscosity of the media, and $r$ is the hydrodynamic radius related to the size of the particle. This means that under defined electrophoretic conditions, small and highly charged molecules will move toward the electrode with the opposite charge faster than bulky and neutral ones. From the perspective of sample treatment, this allows for an active and precise separation of contaminants from the target molecule (given the unique electrophoretic properties of the different biomolecules present in blood/ serum samples), dramatically reducing nonspecific interactions. $^{31-33}$

Electrophoresis can be carried out either in free solution (capillary electrophoresis) or in a support medium (paper or gel). The former offers fast and high-resolution separation but requires strong electric fields, which limits its application at the POC (i.e., expensive and bulky equipment is needed to generate such electric fields, which may also be dangerous for the final, untrained user). The latter is simpler, cheaper, and enables facile visualization of the separation zone by direct staining. Within the types of support media, the gel provides better resolution than paper but is more affected by heat generation, which causes variations in the particle's migration rate and band's distortion. ${ }^{32,34}$ Contrary to free electrophoresis, the particle's movement in support-medium-electrophoresis is a sum of electrophoretic and electroendosmotic mobility. Electroendosmosis is the movement of liquid toward an anode or a cathode depending on the type of support medium, potential gradient, electrolyte ionic strength, and $\mathrm{pH}$. As a consequence, depending on the specific conditions, we could observe charged molecules moving toward the electrode with the same charge when the electroendosmosis forces are greater than the electrophoretic ones. ${ }^{35-37}$

Unexpected particle's mobility or band's distortion is commonly observed upon heat generation during gel electrophoresis. ${ }^{38}$ This phenomenon is explained by Joule's law and the generalized power equation

$$
P=\frac{V^{2}}{R}
$$

where $(P)$ is the power converted from electrical energy to thermal energy, $(V)$ is the voltage applied, and $(R)$ is the resistance to current. ${ }^{39}$ Considering this equation, electrical systems that exert higher resistance to current produce less heat. In this sense, paper electrophoresis is less prone to heat generation than agarose gel electrophoresis because the NC membrane has $10^{9}$-fold higher resistivity than agarose gel and, therefore, it provides higher resistance to current. ${ }^{40,41}$

The core of PEB is based on paper electrophoresis, which since 1950s has been developing strategies for the examination of a variety of different samples, ranging from water to human serum. ${ }^{42-44}$ Most of these works aimed at the separation of target analytes (including inorganic salts, amino acids, enzymes, and proteins) along a filter paper and their subsequent analysis, such as the measurement of their concentration and/or specific activity. ${ }^{45}$ Nonetheless, these works relied on complex and tedious techniques for the identification of the separated components, which are based on staining or immunoblotting. The former serves for an estimation of the molecular size of the components upon comparison with a ladder/marker. The latter provides a more specific determination of the separated components, but it still requires several complex steps (membrane transferring, incubation with a primary antibody, washing, incubation with a secondary antibody, washing, signal revealing) and high volumes of antibodies.

For the development of PEB, we took advantage of the sample treatment capabilities of paper electrophoresis and merged them with the convenience of LFAs. The result is a diagnostic platform that can receive an untreated drop of blood and produce an easy-to-read optical signal in less than $15 \mathrm{~min}$.

Design and Operation of the PEB Device. We designed the PEB device to assure its application at the point-of-care, retaining the portability, usability, and low cost of the LFA. For this reason, we used the standard LFA materials: cellulose and NC membranes. The cellulose pads were used as buffer reservoirs to maintain the system constantly wet and the NC membrane was used as the main platform for carrying out both the electrophoretic separation and the signal generation. We also used two sticky copper electrodes instead of applying the voltage directly on the NC strip. Compared to crocodile clips or similar electrical connectors, the copper sticky tape provides a higher contact surface on the NC strip. In addition, it is cheap and can be disposed together with the paper strip once oxidized (the copper electrode is corroded due to the oxidation reaction when used as an anode during electrophoresis). The paper strip containing the copper electrodes is intended to be disposable once the assay is performed. Regarding the reagents, we used antibodies as bioreceptors (printed on the NC membrane like standard LFA) and analytes, while we employed AuNPs and QDs as labels. To perform the electrophoresis separation, we designed a miniaturized and portable power source as an alternative to the bulky and expensive power supply used in conventional electrophoresis systems. We connected the smartphone battery to a Joule thief inverter and a rectifier (Figure S1) to obtain a stable $200 \mathrm{v} \mathrm{DC}$ $(0.1 \mathrm{~mA}, 20 \mathrm{~mW})$. It is worth mentioning that although the low intensity of the output current may suggest its inadequacy to perform electrophoresis, the high resistance of the system (several megaohms) allows keeping a constant voltage in the device. The paper strip size is $21 \mathrm{~cm} \times 4 \mathrm{~cm}$ (Figure S3), weighs 2.1 grams, and costs around 1 euro (excluding the power source, which costs approximately 10 euros and weighs 10 grams). The assembly procedure of the different pads is similar to that of LFA. ${ }^{18}$ A detailed description of the fabrication of the PEB device is in the Materials and Methods section of this manuscript.

The operation of the sensor consists of few simple steps: (1) the two cellulose pads at the two ends of the sensor are wet 


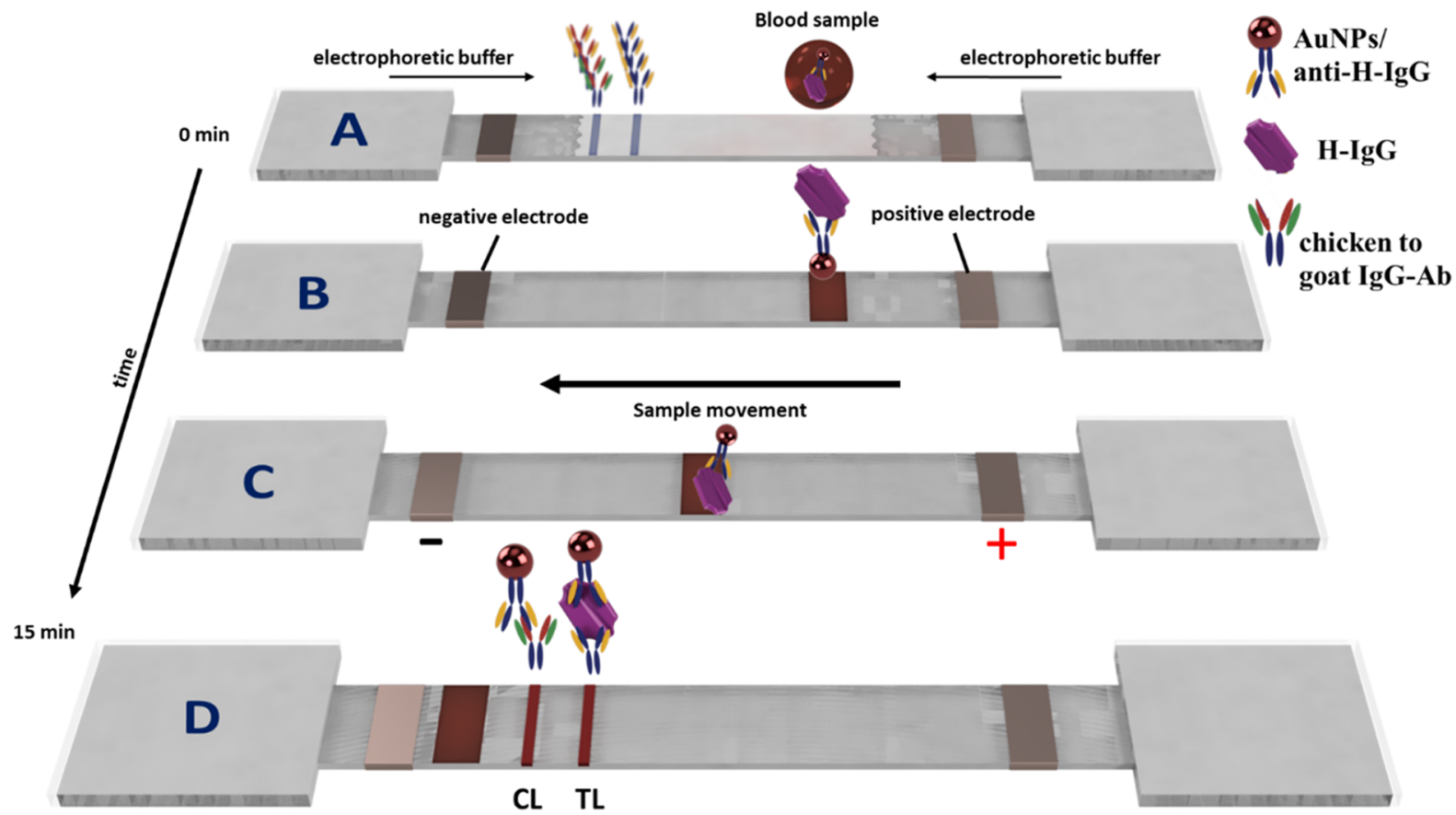

Figure 1. Schematic representation of the PEB strip and assay operation. The PEB strip is composed of an NC membrane strip functionalized with capture antibodies in TL and CL. (A) Two pieces of adhesive copper tape are stacked on both sides of the strip and serve as cathode and anode electrodes. Two pieces of cellulose membrane are stacked on both ends of the strip and serve as electrophoresis buffer reservoirs. The electrophoresis buffer is drop-casted on both cellulose pads and is gradually dispensed to the NC membrane. (B) Conjugate solution is mixed with the whole blood sample and it is drop-casted on the strip. (C) Sample moves by electrophoresis toward the detection zone. (D) Target analyte and the labeled antibodies are captured by the antibodies in TL and CL. The nonspecific interfering components of the whole blood sample flow out of the detection zone, enabling a clear visualization of the signal in TL and CL.
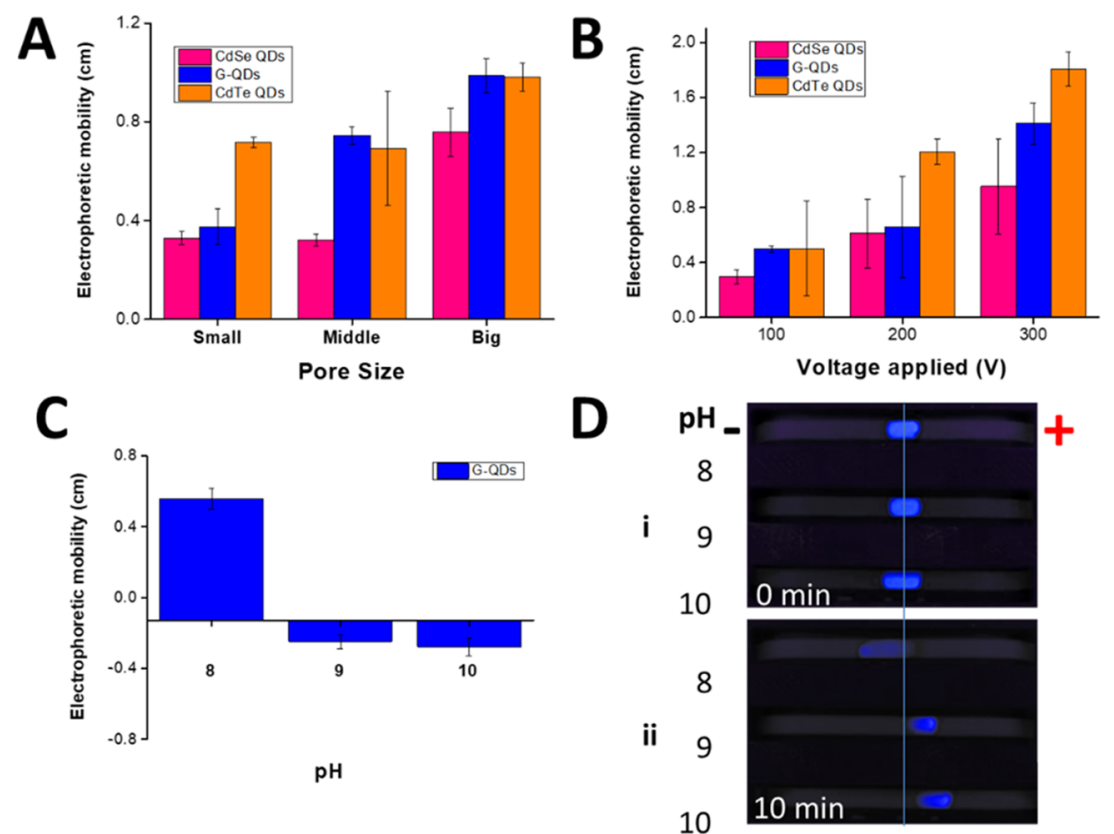

Figure 2. Electrophoresis performance evaluation. (A) Effect of the NC membrane pore size on the electrophoretic mobility of different QDs. NC membranes, CN95, CN110, and CN150, were selected due to their big, medium, and small pore size, respectively. (B) Evaluation of the voltage effect applied on the electrophoresis mobility using the same QDs. (C) Effect of the $\mathrm{pH}$ value on the electrophoretic mobility. Data are shown as mean \pm standard error of mean (SEM) of triplicate experiments. (D) G-QDs image at different $\mathrm{pH}$ values before applying the voltage 0 min (i) and after $10 \mathrm{~min}$ of voltage (ii).

with the electrophoresis buffer, which contains the electrolytes enabling the electric bridge between the cathode and the anode. The high void volume of the two cellulose pads ensures the complete and homogenous wetting of the NC membrane for the whole duration of the assay $(15 \mathrm{~min})$. (2) Once the NC membrane is fully wet (approximately in 2 min from the addition of the electrophoresis buffer), the labeled bioreceptors and sample $(6 \mu \mathrm{L})$ are drop-casted onto the membrane. (3) 
The electrophoretic potential is applied to move the sample along the membrane, which will generate the optical readout. The full operation is schematized in Figure 1. Additionally, a demonstration video using a blood sample where the control line appears after running the electrophoresis assay can be observed in Movie S1. Conversely, Movie S2 shows that there is no movement of the sample when the voltage is not applied (after the paper strip is totally wet with the buffer). Meaning that the sample does not move by capillarity in PEB and only moves when the voltage is applied. Other authors have previously reported the inability of compounds to flow by capillarity in paper when it is soaked and in equilibrium. ${ }^{46}$ It is important to note that, while here we demonstrate the proof of concept of the technology, the entire operation could be condensed into a single-step procedure by keeping the sensor wet in a sealed container and employing a conjugate pad on top of the NC membrane.

Characterization of the PEB as an Electrophoretic Platform. Given the complexity of paper-based electrophoresis, we first characterized how using different materials and reagents during the fabrication of the PEB would affect its electrophoretic behavior. We studied four main components: the particle mobility, the pore size of the NC membrane, the applied voltage, and the $\mathrm{pH}$ of the electrophoresis buffer (Figure 2). We evaluated how the size, shape, and charge of nanoparticles affect their migration speed. According to the concept of electrophoresis, we expect that bigger and less charged particles would move slower than smaller and more charged ones, given a fixed pore size, electrophoretic buffer, and applied potential. To validate this hypothesis, we used three different QDs: CdSe@ZnS QDs functionalized with streptavidin ( $\varnothing 20 \mathrm{~nm}$, considering the streptavidin), CdTe QDs (Ø $5 \mathrm{~nm})$, and G-QDs (Ø $2.5 \mathrm{~nm})$. We chose these three QDs because they cover the size range of most proteins and their charge has been previously studied. G-QDs have a high negative charge due to the oxygen functional groups $(\mathrm{C}-\mathrm{OH}$, $\mathrm{C}=\mathrm{O}$, and $\mathrm{COOH}) ;{ }^{47} \mathrm{CdTe}$ QDs also have a negative charge due to their functionalization with carboxyl groups. ${ }^{48}$ The streptavidin $(\mathrm{pI} \approx 5)$ functionalization of the CdSe@ZnS QDs also confers them a negative surface charge at $\mathrm{pH}$. Their detailed characterization can be found in the Supporting Information (emission spectra, excitation spectra, and transmission electron microscopy (TEM) images are shown, respectively, in Figures S4-S6). For this experiment, we fixed the field strength using $10 \mathrm{mM} \mathrm{SB}$ buffer at $\mathrm{pH} 8$ and an operating voltage of $200 \mathrm{~V}(0.1 \mathrm{~mA}, 20 \mathrm{~mW})$ and the CN150 NC membrane. Despite their negative surface charge, the QDs moved to the cathode (negative electrode) and this was attributed to electroendosmosis, which is the flow of the electrolyte solution. Since the buffer solution has a $\mathrm{pH}$ value lower than the $\mathrm{p} K_{\mathrm{a}}$ value of the boric acid $\left(\mathrm{p} K_{\mathrm{a}}=9.24\right)$, the predominant concentration of the positively charged ions makes the electrolyte solution move toward the cathode, dragging the QDs with it. As expected, we found a correlation between the size/charge and the migration speed. In particular, the CdSe@ZnS QDs moved at an average speed of $0.32 \mathrm{~cm} /$ min toward the negative electrode due to their bigger size $(20$ $\mathrm{nm}$ ) and the negative surface charge provided by the streptavidin $(-60.3 \mathrm{mV})$. However, despite its z-potential value is 3 times higher than the G-QDs one $(-20 \mathrm{mV})$, the bigger size of the CdSe@ZnS QDs $(20 \mathrm{~nm})$ compared to the G-QDs $(2.5 \mathrm{~nm})$ allows the G-QDs to move slightly faster $(0.37 \mathrm{~cm} / \mathrm{min})$. Likewise, despite the 2 -fold bigger size of
CdTe QDs compared to the G-QDs, their higher negative surface charge provided by the carboxyl functional groups $(-56.9 \mathrm{mV})$ makes them move faster $(0.72 \mathrm{~cm} / \mathrm{min})$.

The second parameter that we studied was the effect of different NC membranes over the migration speed. For this study, we used three different NC membranes (CN95, CN110, and CN150) commonly used for the development of LFAs. Particularly, they provide three different capillary flow rates of $95 \mathrm{~s} / 4 \mathrm{~cm}, 110 \mathrm{~s} / 4 \mathrm{~cm}$, and $150 \mathrm{~s} / 4 \mathrm{~cm}$ for CN95, CN110, and $\mathrm{CN} 150$, respectively. The capillary flow rate represents the required time for the sample to cover $4 \mathrm{~cm}$ of the $\mathrm{NC}$ membrane, which is inversely related to the membrane pore size. For example, CN150 with a capillary flow rate of $150 \mathrm{~s} / 4$ $\mathrm{cm}$ has the smallest pore size of the three membranes tested in this work. The fabricants of NC membranes do not give information on the pore size as the polymeric structure does not actually create pores but rather a sponge-like pathway. ${ }^{49}$ We refer the reader to Figure S7 containing the scanning electron microscopy (SEM) images of the three different membranes and the estimation of their relative pore size. Again, the PEB device responded accordingly to the electrophoresis model. For this experiment, we used $10 \mathrm{mM}$ SB buffer at $\mathrm{pH} 8$, an operating voltage of $200 \mathrm{~V}(0.1 \mathrm{~mA}, 20 \mathrm{~mW})$, and the same QDs. As expected, the CN95 membrane produced 2.67-, 1.37-, and 2.30-fold faster migration speed of G-QDs, CdTe, and CdSe@ZnS QDs, respectively, compared to the CN150 membrane (Figure 2A).

The third parameter we tested was the applied voltage. In electrophoresis, under a uniform electric field, the field strength is directly proportional to the voltage applied and hence to the particle's velocity. Therefore, the higher the voltage, the faster the migration speed when the other parameters are fixed. From the engineering point of view of the sensor fabrication and design, this is a crucial parameter since we need to be able to achieve a quick migration applying a relatively low voltage to have a sensor deployable at the point-of-care. In Figure 2B, we show the behavior of the voltage on the electrophoretic mobility at $100(0.05 \mathrm{~mA}, 5$ $\mathrm{mW}), 200(0.1 \mathrm{~mA}, 20 \mathrm{~mW})$, and $300 \mathrm{~V}(0.15 \mathrm{~mA}, 45 \mathrm{~mW})$. For this experiment, we used CN95 NC membrane because this type of NC membrane provided similar speeds for the three QDs (Figure 2A). As expected, a higher voltage caused a faster movement of the QDs to the negative electrode. G-QDs, CdTe, and CdSe QDs moved 2.86, 1.50, and 1.54-times faster when applying $300 \mathrm{~V}$ rather than $200 \mathrm{~V}$. However, higher voltages resulted in less reproducible velocities. The standard deviation of the velocities of G-QDs, CdTe, and CdSe QDs were 2.56, 3.55, and 6.60-times higher when applying $300 \mathrm{~V}$ rather than $100 \mathrm{~V}$. The CdTe QDs were the fastest, particularly they moved 1.3 times faster than the G-QDs when applying $300 \mathrm{~V}$. Instead, the CdSe QDs followed the same trend as in Figure $2 \mathrm{~A}$ and moved slowest to the negative electrode.

Finally, to evaluate the effect of the $\mathrm{pH}$ on the particle mobility direction and speed, we tested SB buffer $(10 \mathrm{mM})$ at three different $\mathrm{pH}$ values $(8,9$, and 10$)$. The particle's rate and direction of movement are influenced by electrophoretic and electroendosmotic mobilities. Variations in the $\mathrm{pH}$ have a strong effect on both forces. In the former, the $\mathrm{pH}$ determines the magnitude and sign of particle's surface charge, while in the latter the $\mathrm{pH}$ determines the rate and direction of movement of the electrolyte solution. Being able to tune not only the speed but also the direction of the flow is paramount to achieve a complete sample treatment. In fact, just by changing the $\mathrm{pH}$ of 
the electrophoresis buffer, we could separate the target analyte from other compounds. For this experiment, we challenged the PEB device with G-QDs since their surface functional groups are deprotonated when the $\mathrm{pH}$ value is higher than $7 .^{50}$ This means that the G-QDs have a higher negative surface charge upon $\mathrm{pH}$ values higher than 7 . However, we observed that at $\mathrm{pH} 8$, the G-QDs moved toward the negative electrode, at $\mathrm{pH}$ 9 they slightly moved, and at $\mathrm{pH} 10$ they moved toward the positive electrode (Figure 2C,D). This means that the mobility of the G-QDs was mostly influenced by the electroendosmotic mobility: at $\mathrm{pH} \mathrm{8,} \mathrm{the} \mathrm{pH}$ value of the buffer was lower than the $\mathrm{p} K_{\mathrm{a}}$ value of the boric acid $\left(\mathrm{p} K_{\mathrm{a}}=9.24\right)$ and, therefore, the predominant ions in the electrolyte solution were positively charged. The buffer dragged the G-QDs to the negative electrode. At $\mathrm{pH} 9$, the $\mathrm{pH}$ value of the buffer was nearly equal to the $\mathrm{p} K_{\mathrm{a}}$ value of the boric acid $\left(\mathrm{p} K_{\mathrm{a}}=9.24\right)$; therefore, there were no predominantly charged ions in the electrolyte solution and the G-QDs had enough negative surface charge to slightly move toward the positive electrode. Conversely, at $\mathrm{pH} \mathrm{10,} \mathrm{the}$ predominant ions in the electrolyte were negatively charged; thus, the buffer dragged the G-QDs toward the positive electrode. Once again, the PEB device was found to adhere to the laws of paper-based electrophoresis.

After demonstrating that the PEB device was responding accordingly using simple homogenous buffer solutions, we verified that our platform is able to separate different particles from a heterogeneous sample. To achieve this, we used two different solutions: one containing $\mathrm{N}-\mathrm{S}$-doped G-QDs and CdSe@ZnS QDs (at $25 \mathrm{nM}$ ), while the other containing CdTe QDs and CdSe@ZnS QDs (both at $25 \mathrm{nM}$ ). The choice of these two couples of QDs gives relevant information on the influence of the surface charge and size on the particle's mobility. For instance, in the previous section, we observed that the G-QDs moved faster toward the cathode than the CdSe@ZnS QDs, mainly due to their big difference in size (2.5 and $20 \mathrm{~nm}$, respectively). However, we wanted to check if this phenomenon could be conversed by manipulating the surface charge of the G-QDs. In this sense, we doped the G-QDs with nitrogen and sulfur functional groups to increase their negative surface charge, which provides repulsion to the cathode. It is reported that the $\mathrm{N}-\mathrm{S}$-doped G-QDs have a more negative $\zeta$ potential value than the G-QDs $(-31.6$ and $-20 \mathrm{mV}$, respectively). ${ }^{47,51}$ The system set up included the use of the SB buffer at $\mathrm{pH} 8$, a potential of $200 \mathrm{~V}(0.1 \mathrm{~mA}, 20 \mathrm{~mW})$, and the CN150 NC membrane. This membrane provides the slowest speeds and, therefore, the mobility progression of the QDs can be evaluated with more detail. Again, the system responded as expected. Both QDs in the mixture moved toward the cathode due to electroendosmosis. However, the N-S-doped G-QDs moved slower than the CdSe@ZnS QDs toward the negative electrode despite their smaller size, indicating that the CdSe@ZnS QDs have less negative surface charges than the N-S-doped G-QDs (Figure 3A). Instead, for the second mixture, the smaller CdTe QDs (diameter $5 \mathrm{~nm}$ ) moved faster than the bigger CdSe@ZnS QDs (Figure 3B), which is similar to what we observed in Figure 3A. Crucially, in both cases, we achieved the complete separation of different QDs in the respective solutions in $10 \mathrm{~min}$. This separation was promoted by the difference in electrophoretic mobility of the QDs and not by electroendosmosis. This result supports the ability of the PEB device to carry out sample treatment for the separation of both particles with different sizes and different charges.
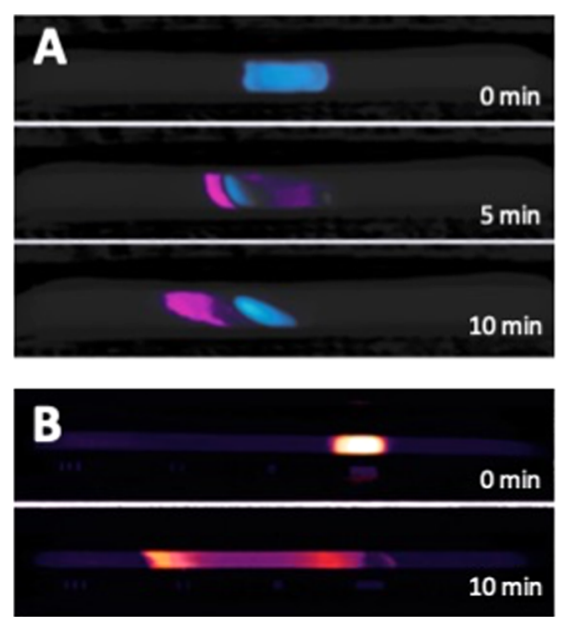

Figure 3. Separation of QDs by electrophoresis. (A) N-S-doped GQDs are mixed with CdSe@ZnS QDs and drop-casted in the center of the NC membrane strip that is immersed in electrophoresis buffer, $\mathrm{pH}$ 8. After running the electrophoresis for $10 \mathrm{~min}$, the CdSe@ZnS QDs (pink) moves to the left side of the strip (negative electrode), separating from the N-S-doped G-QDs (blue), which have slightly moved to the left side. (B) CdTe QDs are mixed with CdSe@ZnS QDs and drop-casted onto the center of the NC membrane strip that is immersed in electrophoresis buffer, $\mathrm{pH}$ 8. After running the electrophoresis for $10 \mathrm{~min}, \mathrm{CdSe} @ \mathrm{ZnS}$ QDs (orange) have moved faster to the left side (negative electrode), separating from the CdSe@ $\mathrm{ZnS}$ QDs (red), which have slightly moved to the left side.

PEB Optimization for Gold Nanoparticles. Supported by the ability of the PEB device to separate different types of QDs, we decided to test its ability to move also AuNPs. Aiming at the development of a POC platform, the use of AuNPs (the most used label in LFA) is fundamental to translate the device from the benchtop to the market since they provide cost-effective synthesis, straightforward functionalization, and naked-eye visualization. ${ }^{52-54}$

The AuNPs have a spherical shape and a diameter of around $15 \pm 2$ and $34.8 \mathrm{~nm}$ when measured in TEM and DLS, respectively. Their $\zeta$-potential value is around $-49.9 \mathrm{mV}$ (the reader can find a complete characterization of the AuNPs in the SI (Figures S8 and S9)). In particular, we evaluated the mobility of both citrate-capped AuNPs and AuNPs functionalized with bovine serum albumin (BSA) and goat IgG (which are the detection reagents used in the final application). The system set up consisted of the use of a CN150 NC membrane, $10 \mathrm{mM}$ SB buffer, $\mathrm{pH} 8$, and a potential of $200 \mathrm{~V}(0.1 \mathrm{~mA}, 20$ $\mathrm{mW})$. The CN150 NC membrane runs at a slower speed compared to $\mathrm{CN} 95$ and $\mathrm{CN} 110$ and thus more time to accurately determine the distance traveled by the functionalized AuNPs. As expected, the citrate-capped AuNPs had higher mobility compared to the biofunctionalized AuNPs. The electrophoretic velocity decreased 1.25-, 1.38-, and 1.51fold upon the functionalization with BSA $(-44.1 \mathrm{mV}, 38.0$ $\mathrm{nm})$, BSA + anti-H-IgG $(-35.2 \mathrm{mV}, 56.3 \mathrm{~nm})$, and BSA + anti-H-IgG + H-IgG (target molecule) $(-27.5 \mathrm{mV}, 58.8 \mathrm{~nm})$, respectively. This behavior is mainly due to the increase of the hydrodynamic diameter of the AuNPs (BSA has an approx. diameter of $3 \mathrm{~nm}$, while IgG of $10 \mathrm{~nm}) .{ }^{55}$ As expected, we observed that the AuNPs moved to the negative electrode due to the movement of the buffer toward the cathode (electroendosmotic force) (Figure S10). ${ }^{35-37}$ 

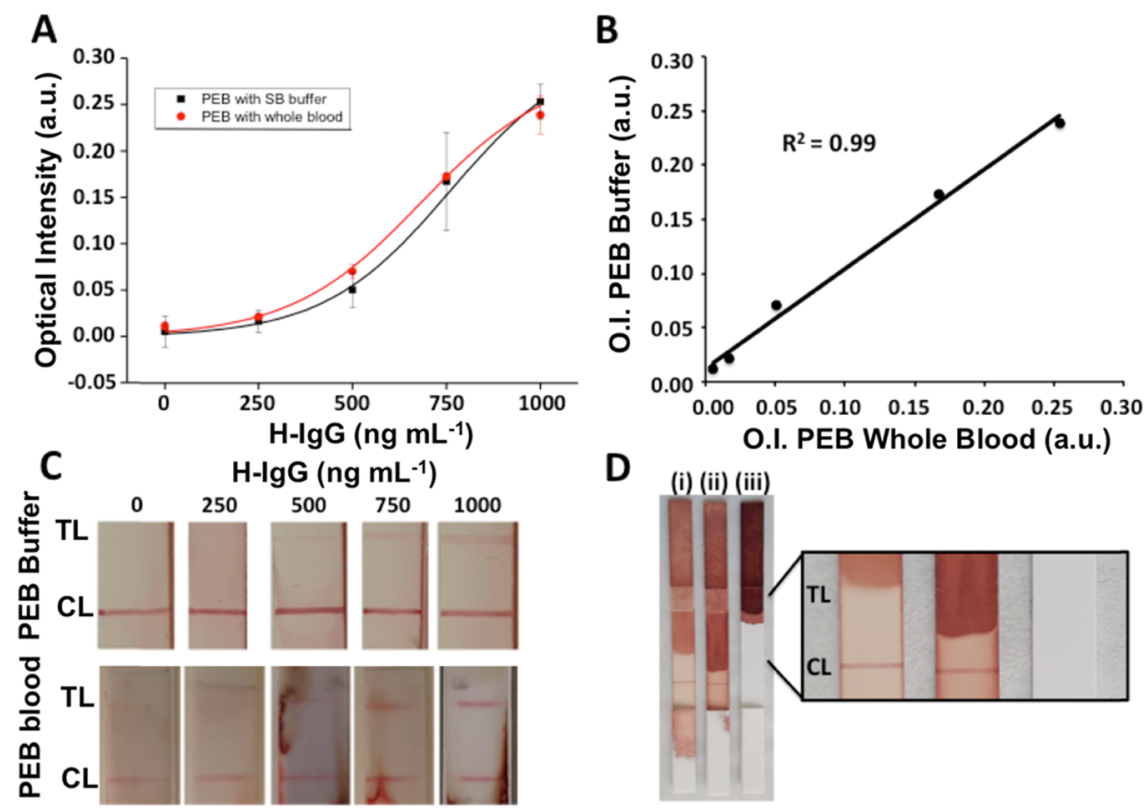

D

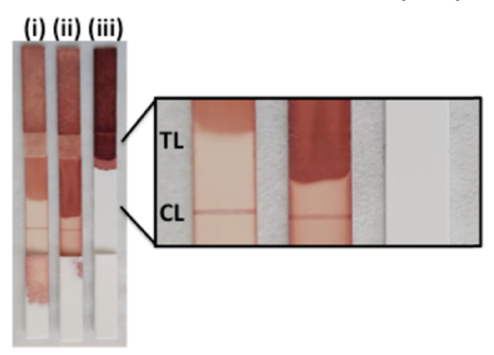

Figure 4. Calibration curve for H-IgG detection using the smartphone camera. (A) Calibration curve with PEB in buffer media (black) and whole blood (red), covering the range from 0 to $1000 \mathrm{ng} / \mathrm{mL}$. The curve equations with PEB in buffer and whole blood were (optical intensity $=2 \times 10^{-7}$ $\left.[\mathrm{H}-\mathrm{IgG}(\mathrm{ng} / \mathrm{mL})]^{2.0248} r^{2}=0.98\right)$ and (optical intensity $\left.=1 \times 10^{-6}[\mathrm{H}-\mathrm{IgG}(\mathrm{ng} / \mathrm{mL})]^{1.7807} r^{2}=0.98\right)$, respectively. (B) Correlation between the signals obtained in PEB when performing the calibration curve $(0-1000 \mathrm{ng} / \mathrm{mL})$ in buffer and whole blood (optical intensity PEB Buffer $=0.9187$, optical intensity PEB Whole blood $\left.+0.0124 r^{2}=0.99\right)$. Data are shown as mean \pm SEM of triplicate experiments. (C) Pictures of PEB strips after performing the calibration curve $(0-1000 \mathrm{ng} / \mathrm{mL})$ in buffer and whole blood. (D) Pictures of LFA strips after the detection of H-IgG (1000 ng/ $\mathrm{mL}$ ) in (i) 5-fold diluted whole blood, (ii) 2.4-fold diluted, and (iii) undiluted whole blood. The inset picture showing a zoom-in on the detection area.

PEB Optimization for Whole Blood Analysis. As we previously described, the type of NC membrane can dramatically affect the performance of the PEB device. For this reason, we tested the ability of CN95, CN110, and CN150 membranes to move whole blood while applying a potential of $200 \mathrm{~V}(0.1 \mathrm{~mA}, 20 \mathrm{~mW})$ in the SB buffer at $\mathrm{pH} 8$. Similar to what is reported in other works, we observed that the flow rate was greater at the anode and decreased toward the cathode. ${ }^{45}$ This effect is related to the equilibrium reached between the electroendosmotic force and the osmotic pressure, two opposite forces leading to the accumulation of liquid nearby the cathode. This means that while the applied potential induces the movement of the electrolyte solution toward the cathode, the accumulation of the electrolyte solution at the cathode creates an osmotic pressure toward the anode until reaching the equilibrium of forces. The result is that the sample stops moving after $15 \mathrm{~min}$. We observed that CN95 was the only NC membrane capable of washing out the blood from the detection zone in $15 \mathrm{~min}$ (see Figure S11). This is according to the electrokinetic theory, stating that bigger pores produce faster mobility. ${ }^{53}$ While a lengthier strip (the equilibrium of forces would be reached later) together with more volume of electrophoresis buffer (to avoid fast evaporation) would enable a longer assay time, we preferred to use a bigger pore (CN95) membrane in the next experiments since it allows the assay to be performed in just $15 \mathrm{~min}$.

Detection of H-IgG in Whole Blood. As the final step toward demonstrating the applicability of the PEB device as a point-of-care diagnostic platform, we employed it for the detection of a biomarker in spiked whole blood. As the test bed, we used a model system previously optimized in our lab for the detection of human IgG. The choice of this analyte has two main reasons: (1) it provides a convenient comparison to
LFAs developed in our lab and (2) the detection of H-IgG in blood represents a real clinical scenario, as shown by the recent pandemic. ${ }^{1,56}$ To facilitate the interpretation of the results and limiting the sample-to-sample variation, we spiked chicken whole blood with $\mathrm{H}$-IgG, in this way, we removed the variability lead by the endogenous concentration of human IgG in human blood, which is in the range of $3-16 \mathrm{mg} / \mathrm{mL} .^{57} \mathrm{~A}$ detailed experimental procedure can be found in the SI section, while the optimization of the preincubation step can be found in Figure S12.

Before going to the analysis of whole blood, we characterized the analytical performance of the PEB device and compared it with the one of LFAs using buffered solutions spiked with H-IgG. We performed a calibration curve in LFA covering the range from 0 to $1000 \mathrm{ng} / \mathrm{mL}$ for the $\mathrm{H}-\mathrm{IgG}$ concentration (values above $1000 \mathrm{ng} / \mathrm{mL}$ usually produces the hook effect, by which we observe a decrease in the signal due to the saturation of the binding domain of the bioreceptors) (Figure S13). ${ }^{58}$ We then performed a calibration curve in PEB covering the same range of H-IgG (Figure 4A). The LoD and LoQ achieved in LFA were 265.56 and $1876.94 \mathrm{ng} / \mathrm{mL}$, while the ones for PEB were 489.78 and $856.09 \mathrm{ng} / \mathrm{mL}$, respectively. These are similar to the publications recently reported by our group. ${ }^{10}$ Besides showing a higher LoD, PEB proved a 2.2-fold lower LoQ and a 4.5-fold higher sensitivity than LFA when comparing the curve slopes (0.44 and 2.02 for LFA and PEB, respectively). It is noteworthy that the strong affinity of the bioreceptors to the target analyte created a stable signal in TL and avoided the dragging of the labeled bioreceptors from the TL by the strong electric field.

Finally, supported by the positive results obtained with buffer solutions, we used the same range of concentrations but using undiluted whole blood (Figure 4A,B). The PEB device 
produced a specific and sensitive response $(\mathrm{LoD}=284.52 \mathrm{ng} /$ $\mathrm{mL}$ and LoQ $=441.07 \mathrm{ng} / \mathrm{mL}$ ). It is noteworthy that PEB showed lower LoD and LoQ when detecting H-IgG in whole blood than in buffer medium. The reason behind this is that the materials and electrophoretic conditions used in PEB have been specifically optimized to achieve the highest sensitivity when analyzing spiked whole blood (details in Materials and Methods). Conversely, buffer medium flow 1.83-fold faster than whole blood when using the optimized conditions for whole blood (see Figures S11 and S12). This has an impact on the assay's sensitivity, which is highly affected by the flow rate (i.e., a faster flow decreases the interaction time between target and bioreceptors)..$^{59}$ Although PEB enabled the detection of $\mathrm{H}$-IgG in whole blood, avoiding, on the one hand, nonspecific interactions in TL for blank samples and, on the other hand, responding accordingly to the increase in the $\mathrm{H}$-IgG concentration (Figure 4C), CN95-based LFA failed in producing a reading signal due to the lack of movement by the blood sample (Figure 4D).

The LFA strips were fabricated following the procedure reported in the materials and methods section, and a commercial cellulose membrane specifically designed for the analysis of whole blood samples was used as the sample pad. The whole blood sample did not flow properly until it was diluted 5-fold in buffer medium, but still the background signal remained high and favored the masking of the red colorimetric signal in TL. In addition, the dilution of the whole blood sample also implied the dilution of the spiked concentration of H-IgG up to $200 \mathrm{ng} / \mathrm{mL}$, which was lower than the LoD. Therefore, the extra dilution of the whole blood sample improved its flow along the strip but was not appropriate from an analytical viewpoint.

\section{FINAL REMARKS AND CONCLUSIONS}

We observed three main forces affecting the movement of particles within the paper-based electrophoretic platform: electroendosmosis, electrophoresis, and osmosis. First, we saw that the mobility of QDs was influenced by electroendosmosis since all QDs moved toward the cathode (in electrolytic cells, the cathode is negatively charged), despite their negative surface charge. We verified this hypothesis by changing the $\mathrm{pH}$ of the electrophoresis buffer: when the $\mathrm{pH}$ was lower than the $\mathrm{p} K_{\mathrm{a}}$ value of the boric acid $\left(\mathrm{p} K_{\mathrm{a}}=9.24\right)$, the movement of the electrolytes toward the cathode represented the major force of the system, also dragging the negative particles toward the cathode. Instead, for $\mathrm{pH}$ close to the boric acid $\mathrm{p} K_{\mathrm{a}}$ value, the absence of the electrolyte's movement made the electrophoresis the main force, directing the negatively charged nanoparticles toward the anode. Obviously, for $\mathrm{pH}$ higher than the $\mathrm{p} K_{\mathrm{a}}$ value, both the electrophoretic and electroendosmosis forces were moving the particles toward the anode. Then, we observed the influence of electrophoresis in the PEB function since factors such as the particle size, particle surface charge, membrane pore size, and applied voltage were enhancing or hindering the particle mobility, following the general principle of electrophoresis. Additionally, we observed the effect of the osmosis limiting the amount of volume that could be displaced.

The PEB demonstrated to perform efficient sample treatment keeping the main characteristics of point-of-care devices. In fact, employing either a portable battery or a smartphone, we managed to perform two key activities: the separation of different types of nanoparticles in less than 10 min and the flow of blood along NC membranes. Regarding the former, the results we obtained indicate that using NC membranes, whose porosity is orders of magnitude higher than the diameter of the nanoparticles generally employed in LFA, the main factor governing the particle movement is their surface charge, rather than their size. We believe that the ability of PEB to purify particles opens a myriad of opportunities for the treatment and detection of different types of biomarkers. Instead, the latter shows how PEB can handle complex and highly viscous samples without the need for extensive dilutions (which would also decrease the amount of target) or extra washing steps (forcing the user to actuate at different times on the same test). In our opinion, this is a game changer within the field of point-of-care sensors, allowing the use of media such as blood, serum, and other viscous solutions in a simpler and more effective way. In addition, although PEB operation is based on electrophoresis, it can deal with biological samples with different $\mathrm{pH}$ values or salt content. The reason behind this is that the electrophoresis buffer (not the sample contents) is the one leading the flow rate and direction of the sample. The almost 3 orders of magnitude difference in volumes used for the electrophoresis buffer $(2000 \mu \mathrm{L})$ and sample $(6 \mu \mathrm{L})$ makes the electrolytes of the buffer prevail over whole blood salts. Moreover, since there is not a significant diffusion between the sample and the buffer during the electrophoresis, the buffer is leading all the forces, and hence, it is the one responsible for the flow rate and direction modulation. In addition, it is reported that the whole blood samples have generally an ionic strength of $0.15 \mathrm{M}^{60}$ and it is also reported that the factors determining protein's mobility in electrophoresis (retardation coefficients $\left(K_{\mathrm{R}}\right)$, free mobilities $\left(Y_{\mathrm{o}}\right)$, and $R_{\mathrm{f}}$ values) are not affected by $\mathrm{NaCl}$ in the samples up to $0.8 \mathrm{M}$. In fact, the main issue with high-concentrated salt samples in electrophoresis is that SDS cannot bind to proteins. ${ }^{61}$ But this is not our case since SDS is not used in PEB. Regarding the $\mathrm{pH}$ of electrophoresis buffer, this should be optimized for any given application. Similarly, blood samples with different hematocrit levels would have a greater influence on analytical variability in LFA than in PEB since in $\mathrm{PEB}$ all of the whole blood samples are removed from the detection zone by electrophoresis.

Finally, we validated the PEB for the detection of H-IgG in buffered medium and spiked whole blood and compared its analytical performance with LFA. PEB proved a 2.2-fold lower LoQ and a 4.5-fold higher sensitivity (when comparing the curve slopes) than LFA for H-IgG detection in buffer media. Moreover, while LFAs were incapable of directly handling undiluted blood, the signals obtained with PEB when detecting $\mathrm{H}-\mathrm{IgG}$ in spiked whole blood showed a correlation of 0.99 with the ones for the detection in buffer samples. Therefore, PEB proved to be capable of maintaining its outstanding analytical sensitivity when evaluating nondiluted and nonpretreated spiked whole blood. While it is noteworthy that the current approach consists of four main steps, these are easy to be condensed into two steps (current switch-on and sample addition). The relying of the PEB on the connection of the electrodes and the power source can be easily re-engineered by the insertion of a power button, whereas the labeling of the sample and the addition of the electrophoresis buffer can be solved by the storage of the buffer in burstable containers, such as the ones reported by Prof. Mc Neil's group, ${ }^{62}$ and the incorporation of a dedicated conjugate pad using dissolvable wax barriers, such as the ones reported by our group. ${ }^{11}$ Most of 
the commercially available LFA kits are based on a two-step operation; thus, we believe that the proposed optimizations of the prototype are compatible with the application of the concept at the point-of-care.

\section{ASSOCIATED CONTENT}

\section{(s) Supporting Information}

The Supporting Information is available free of charge at https://pubs.acs.org/doi/10.1021/acs.analchem.0c04330.

Materials, reagents, and instruments; conjugation protocol of AuNPs with anti-H-IgG antibodies; detection protocol of $\mathrm{H}-\mathrm{IgG}$ in PEB (Figures S1-S13) (PDF)

Demo video of the PEB device (Movie S1) (AVI)

Demo video when voltage is not applied (Movie S2) (AVI)

\section{AUTHOR INFORMATION}

\section{Corresponding Author}

Arben Merkoçi - Nanobioelectronics \& Biosensors Group, Institut Català de Nanociència I Nanotecnologia (ICN2), CSIC and The Barcelona Institute of Science and Technology (BIST), 08193 Barcelona, Spain; Catalan Institution for Research and Advanced Studies (ICREA), 08010 Barcelona, Spain; 이이이.org/0000-0003-2486-8085;

Email: arben.merkoci@icn2.cat

\section{Authors}

Amadeo Sena-Torralba - Nanobioelectronics \& Biosensors Group, Institut Català de Nanociència I Nanotecnologia (ICN2), CSIC and The Barcelona Institute of Science and Technology (BIST), 08193 Barcelona, Spain

Ruslan Alvarez-Diduk - Nanobioelectronics \& Biosensors Group, Institut Català de Nanociència I Nanotecnologia (ICN2), CSIC and The Barcelona Institute of Science and Technology (BIST), 08193 Barcelona, Spain; 이이이.org/ 0000-0002-9876-1574

Claudio Parolo - Nanobioelectronics \& Biosensors Group, Institut Català de Nanociència I Nanotecnologia (ICN2), CSIC and The Barcelona Institute of Science and Technology (BIST), 08193 Barcelona, Spain; (1) orcid.org/0000-00019481-4408

Helena Torné-Morató - Nanobioelectronics \& Biosensors Group, Institut Català de Nanociència I Nanotecnologia (ICN2), CSIC and The Barcelona Institute of Science and Technology (BIST), 08193 Barcelona, Spain

Alexander Müller - Nanobioelectronics \& Biosensors Group, Institut Català de Nanociència I Nanotecnologia (ICN2), CSIC and The Barcelona Institute of Science and Technology (BIST), 08193 Barcelona, Spain

Complete contact information is available at:

https://pubs.acs.org/10.1021/acs.analchem.0c04330

\section{Author Contributions \\ ${ }^{\S}$ A.S.-T. and R.A.-D. contributed equally to this work.}

\section{Author Contributions}

A.S.-T. and R.A.-D. contributed equally to this work. A.S.-T., R.A.-D., and C.P. designed the experiments. R.A.-D. designed and developed the power source. A.S.-T., R.A.-D., and A.M. designed and fabricated the device. A.S.-T., H.T.-M., and A.M. performed the experiments under the supervision of R.A.-D., C.P., and A.M., A.S.-T., R.A.-D., and C.P. wrote the main manuscript. All authors reviewed and approved the final version of the manuscript.

Notes

The authors declare no competing financial interest.

\section{ACKNOWLEDGMENTS}

We acknowledge financial support from the NACANCEL project PCIN-2016-066 (program Euronanomed 2). This work is also funded by the CERCA Program/Generalitat de Catalunya. The ICN2 is funded by the CERCA program/ Generalitat de Catalunya. ICN2 acknowledges the support of the Spanish MINECO for the Project MAT2017-87202-P and through the Severo Ochoa Centers of Excellence Program under Grant SEV2201320295. R.A.-D. acknowledges the financial support from the EU Graphene Flagship Core 3 Project (No. 881603). A.S.-T. acknowledges the Autonomous University of Barcelona (UAB) for the possibility of performing this work inside the framework of Biotechnology Ph.D. Program. C.P. acknowledges Marie Skłodowska-Curie Actions Individual Fellowship, and this project has received funding from the European Union's Horizon 2020 research and innovation program under the Marie Skłodowska-Curie grant agreement No. 795635. We also acknowledge Prof. Artur Xavier Roig from the Veterinary faculty in $\mathrm{UAB}$ for providing whole blood.

\section{REFERENCES}

(1) Udugama, B.; Kadhiresan, P.; Kozlowski, H. N.; Malekjahani, A.; Osborne, M.; Li, V. Y. C.; Chen, H.; Mubareka, S.; Gubbay, J. B.; Chan, W. C. W. ACS Nano 2020, 14, 3822-3835.

(2) Waggoner, J. J.; Pinsky, A. J. Clin. Microbiol. 2016, 54, 860-867.

(3) Yager, P.; Domingo, G. J.; Gerdes, J. Annu. Rev. Biomed. Eng. 2008, 10, 107-144.

(4) Price, C. P. Br. Med. J. 2001, 322, 1285-1288.

(5) Chin, C. D.; Linder, V.; Sia, S. K. Lab Chip 2012, 12, $2118-$ 2134.

(6) Yetisen, A. K.; Akram, M. S.; Lowe, C. R. Lab Chip 2013, 13, 2210-2251.

(7) Quesada-González, D.; Merkoçi, A. Biosens. Bioelectron. 2015, 73, 47-63.

(8) Parolo, C.; Greenwood, A. S.; Ogden, N. E.; Kang, D.; Hawes, C.; Ortega, G.; Arroyo-Currás, N.; Plaxco, K. W. Microsyst. Nanoeng. 2020, 6, 4-11.

(9) Posthuma-Trumpie, G. A.; Korf, J.; Van Amerongen, A. Anal. Bioanal. Chem. 2009, 393, 569-582.

(10) Quesada-González, D.; Sena-Torralba, A.; Wicaksono, W. P.; de la Escosura-Muñiz, A.; Ivandini, T. A.; Merkoçi, A. Biosens. Bioelectron. 2019, 132, 132-135.

(11) Sena-Torralba, A.; Ngo, D. B.; Parolo, C.; Hu, L.; ÁlvarezDiduk, R.; Bergua, J. F.; Rosati, G.; Surareungchai, W.; Merkoçi, A. Biosens. Bioelectron. 2020, 168, No. 112559.

(12) Millipore, M. Rapid Lateral Flow Test Strips Considerations for Product Development; EMD Millipore Corporation: Billerica, MA, USA, 2013.

(13) Brangel, P.; Sobarzo, A.; Parolo, C.; Miller, B. S.; Howes, P. D.; Gelkop, S.; Lutwama, J. J.; Dye, J. M.; McKendry, R. A.; Lobel, L.; Stevens, M. M. ACS Nano 2018, 12, 63-73.

(14) Li, H.; Han, D.; Hegener, M. A.; Pauletti, G. M.; Steckl, A. J. Biomicrofluidics 2017, 11, 1-15.

(15) Gwyn, S.; Mitchell, A.; Dean, D.; Mkocha, H.; Handali, S.; Martin, D. L. J. Immunol. Methods 2016, 435, 27-31.

(16) Kim, J.; Cao, X. E.; Finkelstein, J. L.; Cárdenas, W. B.; Erickson, D.; Mehta, S. Malar. J. 2019, 18, 1-10.

(17) Zhang, Y.; Liu, X.; Wang, L.; Yang, H.; Zhang, X.; Zhu, C.; Wang, W.; Yan, L.; Li, B. Sci. Rep. 2020, 10, No. 9604. 
(18) Parolo, C.; Sena-Torralba, A.; Bergua, J. F.; Calucho, E.; Fuentes-Chust, C.; Hu, L.; Rivas, L.; Álvarez-Diduk, R.; Nguyen, E. P.; Cinti, S.; Quesada-González, D.; Merkoçi, A. Nat. Protoc. 2020, 15, 3788-3816.

(19) Vivid. Plasma Separation Membrane. https://shop.pall.com/ us/en/medical/advanced-materials/diagnostics/vivid-plasmaseparation-membrane-zidgri78lls (accessed Feb 27, 2020).

(20) Ahlstrom-Munksjö. Lateral flow plasma separation pads. https://www.ahlstrom-munksjo.com/products/medical-life-sciencesand-laboratory/lateral-flow-test-pads/plasma-separation-pads/ (accessed Nov 22, 2020).

(21) Lu, Z.; Rey, E.; Vemulapati, S.; Srinivasan, B.; Mehta, S.; Erickson, D.; Health, G. Lab Chip 2018, 18, 3865-3871.

(22) Gong, M. M.; MacDonald, B. D.; Nguyen, T. V.; Nguyen, K.; Van; Sinton, D. Biomicrofluidics 2013, 7, No. 044111.

(23) Tate, J.; Ward, G. Clin. Biochem. Rev. 2004, 25, 105-120.

(24) Nash, M. A.; Waitumbi, J. N.; Hoffman, A. S.; Yager, P.; Stayton, P. S. ACS Nano 2012, 6, 6776-6785.

(25) Dong, Y.; Shao, J.; Chen, C.; Li, H.; Wang, R.; Chi, Y.; Lin, X.; Chen, G. Carbon 2012, 50, 4738-4743.

(26) Dong, Y.; Pang, H.; Yang, H.; Bin; Guo, C.; Shao, J.; Chi, Y.; Li, C. M.; Yu, T. Angew. Chem., Int. Ed. 2013, 52, 7800-7804.

(27) Turkevich, J.; Stevenson, P. C.; Hillier, J. Discuss. Faraday Soc. 1951, 11, 55-75.

(28) Ambrosi, A.; Castañeda, M. T.; Killard, A. J.; Smyth, M. R.; Alegret, S.; Merkoçi, A. Anal. Chem. 2007, 79, 5232-5240.

(29) Álvarez-Diduk, R.; Orozco, J.; Merkoçi, A. Sci. Rep. 2017, 7, No. 976.

(30) Armbruster, D. A.; Pry, T. Clin. Biochem. Rev. 2008, 29, S49S52.

(31) Adamson, N. J.; Reynolds, E. C. J. Chromatogr. B: Biomed. Sci. Appl. 1997, 699, 133-147.

(32) Fritsch, R. J.; Electrophoresis, I. K. Encyclopedia of Food Sciences and Nutrition; Academic Press, 2003; pp 2055-2062.

(33) Stalcup, A. M. Chiral Separations by Capillary Electrophoresis. In Chiral Analysis; Elsevier, 2006; pp 241-275.

(34) Perrett, D. Electrophoresis. In Encyclopedia of Separation Science; Academic Press, 2000; pp 103-118.

(35) Ferencik, M. Preparation of Pure Immunoglobulins. In Handbook of Immunohistochemistry; Springer, 2012; pp 191-196.

(36) Bikerman, J. J. Electric Surface Phenomena. In Surface Chemistry: Theory and Applications; Academic Press, 1958; pp 405408.

(37) Guo, Y.; Li, X.; Fang, Y. Prog. Biochem. Biophys. 1997, 24, 268272.

(38) Zarei, M.; Goharshadi, E. K.; Ahmadzadeh, H.; Samiee, S. RSC Adv. 2015, 5, 88655-88665.

(39) Experts, D. Electricity, Magnetism and Light. In The General Science Compendium; Disha Publications, 2018; pp 39-50.

(40) Takahashi, M.; Takenaka, H. Polym. J. 1983, 15, 625-629.

(41) Kandadai, M. A.; Raymond, J. L.; Shaw, G. J. Mater. Sci. Eng., C 2012, 32, 2664-2667.

(42) Dangerfield, W. G.; Smith, E. B. J. Clin. Pathol. 1955, 8, 132139.

(43) Mabry, C. C.; Karam, E. A. Am. J. Clin. Pathol. 1964, 34, 143152.

(44) Nanthasurasak, P.; Cabot, J. M.; See, H. H.; Guijt, R. M.; Breadmore, M. C. Anal. Chim. Acta 2017, 985, 7-23.

(45) Woods, E. F.; Gillespie, J. M. J. Biol. Sci. 1953, 6, 130-141.

(46) Lan, W. J.; Maxwell, E. J.; Parolo, C.; Bwambok, D. K.; Subramaniam, A. B.; Whitesides, G. M. Lab Chip 2013, 13, 41034108.

(47) Lee, K. H.; Park, H.; Eom, W.; Kang, D. J.; Noh, S. H.; Han, T. H. J. Mater. Chem. A 2019, 7, 23727-23732.

(48) Quevedo, I. R.; Tufenkji, N. Environ. Sci. Technol. 2012, 46, 4449-4457.

(49) Wong, R.l.; Tse, H. Lateral Flow Immunoassay, 1st ed.; Springer, 2009.
(50) Kumawat, M. K.; Thakur, M.; Gurung, R. B.; Srivastava, R. Sci. Rep. 2017, 7, No. 15858.

(51) Zou, S.; Hou, C.; Fa, H.; Zhang, L.; Ma, Y.; Dong, L.; Li, D.; Huo, D.; Yang, M. Sens. Actuators, B 2017, 239, 1033-1041.

(52) Aldewachi, H.; Chalati, T.; Woodroofe, M. N.; Bricklebank, N.; Sharrack, B.; Gardiner, P. Nanoscale 2018, 10, 18-33.

(53) Cordeiro, M.; Carlos, F. F.; Pedrosa, P.; Lopez, A.; Baptista, P. V. Diagnostics 2016, 6, 43.

(54) Quesada-González, D.; Merkoçi, A. Chem. Soc. Rev. 2018, 47, 4697-4709.

(55) González Flecha, F. L. G.; Levi, V. Biochem. Mol. Biol. Educ. 2003, 31, 319-322.

(56) Bedford, J.; Enria, D.; Giesecke, J.; Heymann, D. L.; Ihekweazu, C.; Kobinger, G.; Lane, H. C.; Memish, Z.; don Oh, M.; Sall, A. A.; Schuchat, A.; Ungchusak, K.; Wieler, L. H. Lancet 2020, 395, 10151018.

(57) Cassidy, J. T.; Nordby, G. L. J. Allergy Clin. Immunol. 1975, 55, $35-48$.

(58) Rey, E.; O’Dell, D.; Mehta, S.; David Erickson, P. Anal. Chem. 2017, 89, 5095-5100.

(59) Bishop, J. D.; Hsieh, H. V.; Gasperino, D. J.; Weigl, B. H. Lab Chip 2019, 19, 2486-2499.

(60) Covington, A. K.; Robinson, R. A. Anal. Chim. Acta 1975, 78, 219-223.

(61) See, Y. P.; Olley, P. M.; Jackowski, G. Electrophoresis 1985, 6, 382-387.

(62) Fernández-Sánchez, C.; McNeil, C. J.; Rawson, K.; Nilsson, O. Anal. Chem. 2004, 76, 5649-5656. 